\title{
Efficacy of Lasers in the Treatment of Tobacco Abuse Induced Oral Leukoplakia
}

\section{Kaushik SK*}

Air Force Dental Centre, Jodhpur, Rajasthan, India

*Corresponding author: Kaushik SK, Air Force Dental Centre Ratanada, Air Force Station Jodhpur Rajasthan India 342011

Received: April 29, 2021; Accepted: May 29, 2021; Published: June 05, 2021

\begin{abstract}
Background: Oral leukoplakia is defined as a white patch or plaque, which cannot be characterized clinically or histopathologically as any other disease entity and is recognized as a common precancerous lesion of the oral mucous membrane. Definitive management crucial due to its notoriety for recurrences and malignant transformation depending on the anatomic location, clinical features, degree of epithelial dysplasia and causative factors. High power pulse diode laser has some advantages like less pain, swelling, prevention of metastasis, edema, less bleeding and infection. The prime objective of the study was to evaluate clinically and histopathologically the disease course with six monthly follow up.
\end{abstract}

Methods: Thirty cases who had white lesion in the buccal mucosa with positive history of oral tobacco abuse, clinically diagnosed cases of oral leukoplakia confirmed by histopathology were selected for inclusion in the study and treated by LASER. The outcomes after the clinical and histopathologic determinants were processed with standard statistical tests, the results were derived and findings concluded.

Results: Cases were distributed as per the size and histopathologic report and LASER ablation was administered Recurrence was noticed in 4 cases $(16.6 \%)$ after six months follow up. No case transformed to malignancy during the study follow up period.

Conclusion: Management of oral leukoplakia prevents not only recurrence and malignant transformation, but also postoperative dysfunction. Laser ablation of tobacco abuse induced leukoplakia is a simple effective and safe alternative to conventional surgical local excision with predictable outcome which can be undertaken as a routine OPD day care set up.

Keywords: Oral leukoplakia; Diode LASER

\section{Introduction}

Amongst the multitude of oral soft tissue afflictions, Oral Leukoplakia $(\mathrm{OL})$ is regarded as a controversial oral mucous membrane precancerous epithelial lesion with a relatively common incidence especially amongst tobacco consumers across all age groups. This clinical entity was first cited in scientific literature way back in 1969 and its definition was first suggested by World Health Organization in the year 1978 as a white patch or plaque over oral mucous membrane which cannot otherwise be characterized clinically or pathologically as any other disease entity. The general concept of oral premalignancy has over the recent past few decades metamorphosized in view of the rejection of the general perception that when such lesions are left untreated, they would turn malignant. As a consequence, it has been accepted currently to be defined as "morphologically altered oral mucous membrane tissue in which cancer is more likely to occur than its apparently normal counterpart". In view of exclusion of various similar other white lesions mimicking OL such as oral lichen planus, white sponge nevus, nicotinic stomatitis, leuko-edema etc, in the year 2007, it was agreed by consensus that the name Oral Leukoplakia should be restricted only to clinical definition. An exhaustive appropriate definition was suggested by Van der Waal in the year 2012 seemingly more apt since it involved the aspect of histological validation as "A predominantly white patch or plaque over oral mucous membrane, that cannot be characterized clinically or histopathologically as any other disorder; and that oral leukoplakia carries an increased risk of development of cancer either in oral cavity or the head and neck region" [1]. Even though the etiology of OL is considered to be multifactorial, indiscriminate long-term tobacco abuse is often an important frequently attributable factor. Carcinogenic toxic substances collectively termed as tar are exceedingly present in content in tobacco. Studies have shown that more than eighty percent of oral leukoplakic patients were consumers of tobacco in some or the other numerous forms with smokers having an amplified risk of developing these lesions. Larger multiple diffuse lesions over different geographic regions of oral mucous membrane were found to be existing amongst frequent highly habituated smokers than those who smoked less. It is fairly apparent from current studies that have steadily shown that early small to medium sized lesions either diminish or become smaller and rarely disappear on cessation of the habit [2-4]. Amongst the numerous substance abuse and habituation, chewing and smoking tobacco have become an alarmingly serious common ill habit across socio economic classes in India. Tobacco was an imported product 
introduced in this country by the Portuguese sea faring explorers and traders nearly four centuries ago and since then it has rapidly become an integral part and parcel of socio economic and cultural identity of various communities across the country irrespective of the ethnic and geographic divide. The impact of tobacco on both urban and rural Indian population has ever since progressed alarmingly so much so that India is now considered the second largest producer and consumer of tobacco next only to China. Historically tobacco was introduced initially as a product only to be smoked, but progressively began to be popular and addictive used in several other forms such as beedi smoking, hookah smoking, chillum pipe smoking, reverse smoking, dry tobacco chewing, processed areca-nut paan masala (betel quid) chewing, gul, ghutka, khaini, mishri, mawa and leaf tobacco chewing. The oral lesion may occur at the anatomic site of the quid placement because of either the direct chemical action of the quid or physical traumatic effect of chewing or both with a tendency for the oral mucosa to desquamate and peal. Alcohol conventionally is attributed to be an important independent risk factor for oral leukoplakia however conclusive data in support of such hypothesis are evidently lacking [5]. Further there are conflicting reports of various studies related to possible causal and casual relationship of Human Papilloma Virus (HPV) infection as an etiology of developing Oral Leukoplakia [6]. OL can look similar to multiple other lesions, moreso in a case such as a mucosal irritation from a dental restorative filling material with mechanical insult, in which case, removal of the offending stimulus and re- evaluation after four weeks generally returns mucosal architecture to normalcy. Definitive treatment of oral leukoplakia for complete removal is very important because of its notoriety for recurrences and possible malignant transformation depending upon the anatomic location, clinical progress, degree of epithelial dysplasia and various etiological factors. Over the last few decades, numerous modalities of treatment both invasive surgical and conservative non-surgical options with medications and combination of the same have been suggested $[7,8]$. Various Invasive surgical options cited in literature for the treatment of this lesion include conventional scalpel wide local excision, ablative monopolar electrocautery, freezing temperature cryosurgery, various classes of laser surgery etc. Conventional surgery may result in side effects of scarring, wound contraction and strictures, and mimicking signs of recurrence [9]. Amongst various laser ablation techniques like $\mathrm{Nd}$ Yag, $\mathrm{CO}_{2}$ laser etc, high energy pulse Diode laser have some added advantages like less pain, swelling, prevention of seeding metastases, edema, minimal intra op bleeding with near dry field and negligible infection when compared to traditional surgical intervention. Presently there are no data of prospective studies for the outcomes after LASER application in the management of oral leukoplakia, both by clinical and histopathological follow up in current literature [1012]. This study was proposed to determine the efficacy of high energy diode LASER in the management of oral leukoplakia with the prime objective to evaluate clinically and histopathologically the disease course with six monthly follow up. The scope of the study rests in the fact that the geographic location of the unit at North Indian state of Uttar Pradesh has a sizeable population traditionally endemic to oral tobacco abuse in common households and entitled clientele reporting with a high incidence of leukoplakia.

\section{Material and Methods}

The Unicentric, interventional, observational, descriptive, longitudinal, prospective study of a cohort of tobacco related Oral Leukoplakia case series managed from Jan 2019 to Jun 2020 was undertaken at a tertiary care specialty health care facility in a geographic belt of country endemically known for excessive tobacco abuse in variety of forms. The study involved screening of all the patients reporting to the centre and selection of those who had white lesion in their buccal mucosa with positive history of oral tobacco abuse. Along with the routine case history, a comprehensive record of the habit history including form, frequency, chronicity, length of contact of tobacco, etc as well as clinical details of the lesion as to site, size, color, consistency, texture, tenderness, etc were recorded. Subjects were counseled \& explained in detail about possible malignant behavior of leukoplakia. The selected subjects were requested to completely refrain from tobacco consumption habit \& were administered oral antioxidants preparation for one month duration prior to subjecting to laser therapy. Those patients whose condition improved to conservative management by reduction of the lesion were excluded from study while those who did not show any response were included for complete laser ablation of lesion. Further any patient on long term medications for any other chronic debilitating diseases were also excluded from the study. The modified classification system for oral leukoplakia suggested by van der Waal et al 2000 in which the size of the leukoplakia and the presence or absence of epithelial dysplasia was taken into account for evaluation and inclusion of the subject into the study [13]. Thirty clinical cases of oral leukoplakia in the buccal mucosa confirmed by histopathology were selected for inclusion in the study and treated by high energy diode LASER after obtaining written informed consent and compliance to abstain from habit during the entire course of the study. The patient informed consent form was obtained and the complete surgical procedure was explained to the patient and prior to performance of surgery.

\section{Surgical procedure}

Complete aseptic conditions were maintained as per standard surgical operating room protocol subsequent to scrubbing and draping after thorough anti septic mouth wash rinse for two minutes. Subsequently local infiltration with $2 \mathrm{cc}$ of $2 \%$ Lignocaine with 1:1,00,000 Adrenaline was given around the lesion. Protective eye goggles were worn by patient, surgeon and assistant. The lesion was painted with Toluidine blue stain. After five minutes, the area where there was increased uptake of stain was excised using $980 \mathrm{~nm}$ Zolar Photon Plus 10 Watt diode laser. The cases were reviewed after one week, fortnight, one month, three months and six months. The clinical subjective signs were noted for improvement/ deterioration or status quo as well as objectively of the dimensions of the lesion, color, consistency, texture etc. Standard Visual Analog Scale (VAS) was used for subjective assessment of postoperative pain, and the clinical healing of lesion was measured as per parameters given in Healing index suggested by Landry et al. [14]. A repeat biopsy was undertaken after six months at the same representative area. The outcomes after the clinical and histopathologic determinants were processed with standard statistical tests, the results were derived and findings concluded.

\section{Results}

A total of hundred patients were screened during the study period 
Table 1: Age and Sex distribution of subjects.

\begin{tabular}{|c|c|c|c|c|}
\hline Sex/Age & $\mathbf{2 0 - 2 9}$ & $\mathbf{3 0 - 3 9}$ & $\mathbf{4 0 - 4 9}$ & $\mathbf{5 0}^{+}$ \\
\hline Male & 2 & 7 & 9 & 4 \\
\hline Female & 0 & 3 & 3 & 2 \\
\hline
\end{tabular}

Table 2: Form of Tobacco habituation.

\begin{tabular}{|c|c|c|c|}
\hline Sex/Tobacco Form & Smokeless & Smoking & Both \\
\hline Male & 12 & 6 & 4 \\
\hline Female & 7 & 0 & 2 \\
\hline
\end{tabular}

out of which thirty $(\mathrm{N}=30)$ were randomly selected for inclusion in the study. The data were collected concurrently during management regarding the parameters and the observations were subject to statistical analysis. Amongst 30 selected leukoplakia cases $22(73.3 \%)$ were males and 8 (26.7\%) were Females; Age ranged from 26 years to 64 years $(42.33+/-4.66)$ (Table 1$)$. The form of consumption of tobacco for habituation showed greater predilection for women towards smokeless tobacco whereas amongst males involved both smoking or smokeless forms including a portion of men indulging in both concurrently (Table 2). Clinical size description wise distribution was $6(20 \%)$ cases L1 (lesion less than $2 \mathrm{~cm}$ in greatest diameter), 14 (46.6\%) cases were L2 (lesion with size between $2-4 \mathrm{cms}$ in greatest diameter), $8(26.6 \%)$ cases were L3 (more than $4 \mathrm{cms}$ in greatest diameter and $2(6.6 \%)$ cases were Lx (area was diffuse with no definite geometric measurable surface. There were total four cases (13.3\%) of recurrence amongst the thirty subjects with $0 / 6(0 \%)$ in $\mathrm{L} 1,1 / 14(7 \%)$ in $\mathrm{L} 2,2 / 8(25 \%)$ in $\mathrm{L} 3$ and $1 / 2$ (50\%) in Lx cases (Table 3). After preliminary confirmatory biopsy the cases were distributed as: 19 (63,3\%) were P0 (No epithelial dysplasia), 9 (30\%) were P 1(distinct epithelial dysplasia) and $2(06.6 \%)$ were Px (dysplasia not specified in report) as determined histopathologically after preliminary biopsy. Recurrence that was noticed in 4 cases (13.3\%) after six months follow up biopsy and histopathological examination revealed 1 out of 19 (5.26\%) had recurrence in P0 cases whereas 3 out of 09 cases (33.33\%) in P1 cases whereas no recurrence was found in

Table 3: Clinical description wise distribution and recurrence

\begin{tabular}{|l|l|l|l|l|}
\hline Size of the Oral Size of OL Size of OL is & Size of OL & Size of lesion is
\end{tabular} Leukoplakia is $<2 \mathrm{~cm} \mathrm{L1} 2-4 \mathrm{~cm} \mathrm{L2}$ is $>4 \mathrm{~cm} \mathrm{L3}$ not specified Lx

\begin{tabular}{|c|c|c|c|c|}
\hline Cases & 6 & 14 & 8 & 2 \\
\hline Recurrences & 0 & 1 & 2 & 1 \\
\hline
\end{tabular}

Table 4: Histopathological distribution and recurrence.

\begin{tabular}{|c|c|c|c|}
\hline $\begin{array}{c}\text { Histopathology of } \\
\text { the lesion }\end{array}$ & $\begin{array}{c}\text { No epithelial } \\
\text { dysplasia P0 }\end{array}$ & $\begin{array}{c}\text { Distinct epithelial } \\
\text { dysplasia P1 }\end{array}$ & $\begin{array}{c}\text { Dysplasia not } \\
\text { specified Px }\end{array}$ \\
\hline Cases & 19 & 9 & 2 \\
\hline Recurrences & 1 & 3 & 0 \\
\hline
\end{tabular}

Table 5: Visual Analog Scale to determine pain after procedure.

\begin{tabular}{|c|c|c|c|}
\hline VAS Score & 7 Days & 15 Days & 1 Month \\
\hline 5 & 3 & 0 & 0 \\
\hline 4 & 8 & 0 & 0 \\
\hline 3 & 15 & 3 & 0 \\
\hline 2 & 2 & 7 & 1 \\
\hline 1 & 2 & 20 & 1 \\
\hline 0 & 0 & 2 & 28 \\
\hline
\end{tabular}

Table 6: Healing Index after procedure.

\begin{tabular}{|c|c|c|c|c|c|}
\hline Healing Index & 1 Week & 15 Days & 1 Month & 3 Months & 6 Months \\
\hline 2 & 6 & 2 & 0 & 0 & 0 \\
\hline 3 & 10 & 8 & 4 & 0 & 0 \\
\hline 4 & 14 & 17 & 4 & 4 & 4 \\
\hline 5 & 0 & 3 & 22 & 26 & 26 \\
\hline
\end{tabular}

Px cases (Table 4). No case transformed to malignancy during the study follow up period. Visual analog scale for post surgical pain evaluation showed almost complete painless recovery between one week to fortnight in all except in two cases at the end of one month. The scores for the cases where residual pain persisted was minimal to mild (Table 5). Post- surgical wound healing as per parameter suggested by Landry et al. showed complete healing at the end of six months in almost all except four cases which were the ones in whom recurrence was noted (Table 6).

\section{Discussion}

The review of current published medical literature is flooded with deep controversies and contradictions regarding various aspects of oral leukoplakia with topics of interest ranging from genetic predisposition, etiological factors, clinical course, histopathological diagnosis, methods of comprehensive management options and their outcome, factors associated with malignant transformation, prognosis, and clinical follow-up. Oral mucous membrane constantly traumatised by various forms of habitual tobacco abuse, as in subjects of this present study might be swiftly and defensively turning over undeveloped or under developed mucosal epithelial cells that are further permeable to carcinogens. This insult is due to the chemical components of tobacco that may directly irritate and stimulate the epithelial keratinocytes and or have an inducive effect by activating cells in the adjoining connective tissue. The accelerated hyperplastic and hyperkeratotic activity often seen in OL represents a rudimentary defensive reaction of the tissue to thwart the noxious stimuli. Various researchers from numerous studies have projected OL to be cautiously dealt as a potentially malignant disease entity with a worldwide prevalence ranging from $0.5 \%$ in a few studies to as high as $3.4 \%$ in some studies. In a prospective longitudinal study undertaken by A Kumar et al, the estimated point prevalence of OL in their study population was reported as $2.6 \%$ (95\% Confidence of Interval 1.72 2.74 ) and they also reported an estimated malignancy conversion rate extending from $0.13 \%$ to as high as $17.5 \%$ [9]. Conventional incisional biopsy with scalpel and histopathological evaluation of the suspicious tissue is still the gold standard for diagnosis. A multitude of variables such as age, association of risk factors, site, size and type of lesion, level of dysplasia, DNA content, have been attributed towards increased risk of carcinogenic change, but no obvious sole reliable biologically active marker has been successful in validation as efficiently and effectively predictable. The yearly malignant conversion rate of oral leukoplakia fluctuates unequally in different parts of the world, due to the type, form, content and frequency of exposure along with environmental and dietary habits as well as other confounding factors and biases. Even though on histopathological examination presence of epithelial dysplasia is an important predictive factor for carcinogenic transformation, it should be realised that not all dysplastic lesions transform to malignancy. On the other hand, sometimes non- 
dysplastic lesions may also sometimes turn malignant. The complete immediate termination of offending mucosal onslaught from oral tobacco habit in any form, being the commonest aetiological agent for oral leukoplakia, has been shown to be a single effective measure with regard to the prevention of incidence of leukoplakia and thereby, the incidence of oral squamous cell carcinoma as well [15]. A plethora of treatment procedures for leukoplakia have been reported with controversial outcomes claiming disappearance of lesion however, there is no consensus of the most appropriate remedy. Interestingly, sometimes, even after successful intervention and complete resection, using various modalities, cases of oral leukoplakia have shown recurrence and or even carcinogenic transformation. Furthermore, new lesions may start to develop in other locations of the oral cavity amongst few patients. Randomised control trials subsequent to nonsurgical intervention do not show any substantiate evidence of effective prevention of malignant transformation or recurrence. Conventional scalpel surgery has its own restrictions especially with respect to the diffuse size and anatomical site of the lesion but few workers have reported laser surgery producing encouraging results. In view of the mysterious nature of the disease progress, there is no universal consensus concerning the length interval or duration period of follow up of such patients $[9,16]$. The Medical usage of Light Amplification by Stimulated Emission of Radiations (LASER) has gained popularity as an effective modality of treatment in various afflictions in medicine and dentistry. Laser ablation surgery for variety of oral conditions has been reported to have multiple advantages, and is nowadays widely used in the management of patients with oral leukoplakia [11]. Post surgical pain after laser ablation of lesion was satisfactory in almost all but two cases at the end of one month. The reduction of VAS values of pain over one month following laser excision of lesion was statistically significant with a p-value of 0.05 . Healing of the laser ablated lesion site was satisfactory at the end of six months in all except four cases in whom were recurrence was noted. The comparative evaluation of wound healing following laser excision of oral leukoplakia was statistically significant with a p-value of 0.05 . In previous studies, recurrence and malignant transformation from the lesion have occasionally been observed following laser surgery. Various authors in published literature have reported the rate of lesion relapse widely ranging from $7.7 \%$ to as high as $38.1 \%$, while malignant change was reported as $2.6 \%$ to $9 \%$ for cases of oral leukoplakia managed by laser surgery. In a similar study by Ishi et al who studied the behaviour of oral leukoplakia reported 29.3\% relapse and $1.2 \%$ carcinogenic transformation [17]. Galletta $\mathrm{V}$ et al, in a more recent study compared high energy pulse diode lasers with Carbon Dioxide lasers and recorded a relapse of the lesion as high as $27.5 \%$ with ten percent developing newer lesions and five percent amongst the cases turning malignant over a mean post intervention observation period of almost two years [18]. In the present study the recurrence was observed in 4 cases (13.3\%) and there was no transformation into malignancy in any of the treated cases during the six months post ablation period. It was observed that the recurrence rate was higher in subjects with lesions of diffuse distribution and size of 2-4 cms or more. Histopathologically the recurrence rate was more when the lesions were reported initially with distinct dysplasia. This differential reporting of lesion size wise and with six month post surgical biopsy and histopathologic relook has not been reported in literature. However due to the unpredictable nature of the lesion for transformation to malignancy all the subjects were advised for further regular periodic preventive oral examination.

\section{Conclusion}

The role of primary health care practitioner either dental or medical professional is quintessential in the early recognition and diagnosis when Oral Leukoplakia lesion is usually inconspicuous and asymptomatic and it is relatively simple and priceless to remove possible etiological factors involved in the initiation especially tobacco consumption in any form, thus reducing carcinogenic transformation too. The chief objective of management of leukoplakia is towards prevention of such malignant transformation. Since there is no absolutely successful satisfactory treatment for leukoplakia, it is best removed in totality and such patients should be regularly monitored for mucosal change. Further they are to be instructed to avoid and refrain from the major risk factors of oral epithelial dysplasia, especially tobacco abuse in any form. Management of oral leukoplakia prevents not only recurrence and malignant transformation but also post-operative dysfunction and LASER surgery is an excellent option as a management procedure to overcome them. It is highly important to inform patients with oral leukoplakia that their condition can be treated safely and effectively by surgical laser which will also be helpful in bringing the odds of cancerous transformation of the lesion. In addition, the patients should also be brought to notice of the attention of necessity of a continued professional monitoring and surveillance regardless of the outcome following surgical intervention. The study concluded that $10 \mathrm{~W}$ Diode laser with the wavelength of $980 \mathrm{~nm}$ can be used in the treatment of oral leukoplakia with no to minimal pain, excellent hemostasis and wound healing with no postoperative complications. Considering the small sample size used in the present study, similar study may be necessary to confirm the results of our study conclusively. High energy Diode Laser ablation of tobacco abuse induced leukoplakia is a simple effective and safe alternative to conventional surgical local excision with predictable outcome which can be undertaken at peripheral primary dental facilities as a routine OPD day care procedure.

\section{References}

1. van der Waal I, leukoplakia O. The ongoing discussion on definition and terminology. Med Oral Patol Oral Cir Bucal. 2015: 20: e685-e692.

2. Chung $\mathrm{CH}$, Yang $\mathrm{YH}$, Wang TY, Shieh TY, Warnakulasuriya S. Oral precancerous disorders associated with areca quid chewing, smoking \& alcohol drinking in southern Taiwan. J Oral Pathol Med. 2005; 34: 460-466.

3. Schepman KP, Bezemer PD, van der Meij EH, Smeele LE, van der Waal I. Tobacco usage in relation to the anatomical site of oral leukoplakia. Oral Dis 2001; $7: 25-27$.

4. Freitas MD, Blanco-Carrión A, Gándara-Vila P, Antúnez-López J, GarcíaGarcía A, Gándara Rey JM. Clinicopathologic aspects of oral leukoplakia in smokers and nonsmokers. Oral Surg Oral Med Oral Pathol Oral Radiol Endod. 2006; 102: 199-203.

5. Hashibe M, Sankaranarayanan R, Thomas G, et al. Alcohol drinking, body mass index and the risk of oral leukoplakia in an Indian population. Int. J. Cancer.2000; 88: 129-134.

6. Bhargava A, Shakeel M, Srivastava AN, Raza TS, Rizvi S, Varshney P. Role of human papilloma virus in oral leukoplakia. Indian J Cancer. 2016; 53: 206209.

7. Ribeiro AS, Salles PR, da Silva TA, Mesquita RA. A review of the nonsurgical treatment of oral leukoplakia. Int J Dent. 2010; 2010. 
8. Awadallah M, Idle M, Patel K, Kademani D. Management update of potentially premalignant oral epithelial lesions. Oral Surg Oral Med Oral Pathol Ora Radiol. 2018; 125: 628-636.

9. Kumar A, Cascarini L, McCaul JA, Kerawala CJ, Coombes D, Godden D, Brennan PA. How should we manage oral leukoplakia? Br J Oral Maxillofac Surg. 2013; 51: 377-383.

10. Schoelch ML, Sekandari N, Regezi JA, Silverman S. Laser management of oral leukoplakias: a follow-up study of 70 patients. Laryngoscope 1999; 109 : 949-953.

11. Ishii J, Fujita K, Komori T. Laser surgery as a treatment for oral leukoplakia. Oral Oncol. 2003; 39: 759-769.

12. Van der Hem PS, Nauta JM, van der Wal JE, Roodenburg JL. Results of CO laser surgery in patients with oral leukoplakia: a 25 year follow up. Oral Oncol. 2005; 41: 31-37.

13. Van der Waal I, Schepman KP, van der Meij EH. A modified classification and staging system for oral leukoplakia. Oral Oncology. 2000; 36: 264-266.
14. Masse JF, Landry RG, Rochette C, Dufour L, Morency R, D'Aoust P. Effectiveness of soft laser treatment in periodontal surgery. Int Dent J. 1993; 43: 121-127.

15. Warnakulasuriya S, Ariyawardana A. Malignant transformation of ora leukoplakia: a systematic review of observational studies. J Oral Pathol Med. 2016; 45: 155-166

16. van der Waal I, Schepman KP, van der Meij EH, Smeele LE. Oral leukoplakia: a clinicopathological review. Oral Oncol. 1997; 33: 291-301.

17. Ishii J, Fujita K, Munemoto S. Management of oral leukoplakia by laser surgery: relation between recurrence and malignant transformation and clinicopathological features. J Clin Laser Med Surg. 2004; 22: 27-33.

18. Galletta V, Azevedo L, Lodi G, Migliari D. Factors affecting Clinical Outcomes after Treatment of Oral Leukoplakia with $\mathrm{CO}_{2}$ and Diode Laser. J Contemp Dent Pract. 2017; 18: 775-780. 